\title{
Clinical pharmacoepidemiology of antibiotics usage in intensive care unit of cancer special hospital
}

\author{
Akrom $^{1,2}$, Rafiastiana Capritasari ${ }^{3}$ \\ ${ }^{1}$ Pharmacology and Clinical Pharmacy Department, Universitas Ahmad Dahlan, Indonesia \\ ${ }^{2}$ Ahmad Dahlan Drug Information and Crisis Center, Indonesia \\ ${ }^{3}$ Poltekes TNI AU Adisucipto, Indonesia
}

\begin{tabular}{l}
\hline \hline Article Info \\
\hline Article history: \\
Received Apr 4, 2020 \\
Revised Jun 11, 2020 \\
Accepted Jun 21, 2020 \\
\hline
\end{tabular}

Keywords:

Antibiotic

Cancer

ICU

Pharmacoepidemiology

Sepsis

\begin{abstract}
The pattern of antibiotics use in cancer patients in the intensive care unit (ICU) of dharma is cancer special hospital (DCSH) has not been identified. The purpose of this study is to determine the pattern of antibiotics use in cancer patients treated in the ICU of DCSH from 2012-2014. This study was observational with a cross-sectional design. The data collection is done retrospectively. The inclusion criteria to recruit the subjects, i.e. Adult patients who had nosocomial infections in the ICU; Patients with medical records in the ICU who received antibiotics in the 2012-2014 periods. Patients originating from inpatients (wards); Patients with medical records were read. The exclusion criteria were postoperative patients and Patients with incomplete medical records. We collected data from medical records of cancer patients who had been admitted to the ICU in 2012-2014, medication administration records, sample submission, and laboratory records. There are 202 cancer patients including in the study. Leukemia and breast cancer were the most cancer's diagnosis in the Subject. More than two hundred cancer patients were receiving antibiotic therapy, with more than $50 \%$ of them were diagnosed with pneumonia, followed by central infection $(>20 \%)$ and urinary tract infection $(>10 \%)$. The antibiotic most frequently used was meropenem, with $33.8 \%$. The three most commonly used antibiotics from 2012 to 2014 were meropenem, levofloxacin, and ceftriaxone.
\end{abstract}

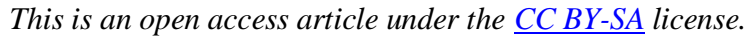

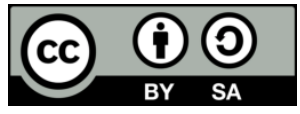

Corresponding Author:

Akrom,

Pharmacology and Clinical Pharmacy Department,

Universitas Ahmad Dahlan,

Yogyakarta, Indonesia.

Email: akrom@pharm.uad.ac.id

\section{INTRODUCTION}

Infection contracted in the hospital intensive care unit (ICU) is one of the health problems in the world and Indonesia [1, 2]. Research in 11 Indonesian hospitals in 2004 showed that the incidence of nosocomial infections was $9.8 \%$. A similar study in 2005-2010 showed that the incidence of hospital care-associated infection (HAI) in Indonesia was lower at 7.1\% [3]. However, the figure was still higher than in China, where the incidence was reported at 3.6\% [4]. Patients treated in the ICU generally have a higher risk of infections with particular bacterial patterns [5,6]. Cancer patients have a higher risk of infections while undergoing treatment in hospitals, especially in ICU(2). Pathological conditions and chemotherapy weaken their immunity so that they are more at risk for infection when they are admitted to ICU. Research in DCSH in 2011-2012 in 119 cancer patients treated with a central venous catheter (CVC) shows that $18.5 \%$ of patients were diagnosed with sepsis, $47.9 \%$ with colonization, and $14.3 \%$ with bacteremia. 
The incidence of bloodstream infection related CVC (BSI-CVC) was $13.3 \%$, which occurred mostly in patients with hematological malignancies (78.4\%) and in impaired-immunity isolation room (90\%) [3]. Other factors associated with increased risk of infection in ICU patients with cancer include the use of invasive health devices such as ventilators and the treatment length [7]. Diseases commonly contracted by patients in the ICU are urinary tract infections [8], lower respiratory tract infections [9], and infections due to the catheter or central venous infusion use [3,10]. Until now, antibiotics are still the primary weapon to overcome the incidence of infection in the ICU, but the issue of germ resistance to antibiotics raises the fear and anxiety of many clinicians. Hospital management requires data on the pattern of antibiotic use for policymaking and anticipating resistance events.

Antibiotics are the first drugs to deal with and prevent the incidence of bacterial infections in the hospital, including in the ICU [11-13]. The extent of bacterial resistance to antibiotics in hospitals, especially in the ICU, has been widely reported $[14,15]$. In the ICU, the resistance tends to occur more quickly because of treatment duration and immune system impairment $[5,16]$. Several types of antibiotics are reported to have resistance events for use in ICU [17, 18]. Antibiotic resistance provides a broad impact both for patients, hospitals, and the government. Monitoring antibiotic use patterns is one method of early detection of antibiotic resistance events. Data on the use of antibiotic patterns from time to time is one important information to predict trends in the incidence of antibiotic resistance in the ICU $[19,20]$. Still, until now, the pattern of antibiotic use in ICU for cancer patients is not enough available in Indonesia. The purpose of this study is to determine the pattern of antibiotic use in cancer patients in the ICU of DCSH.

\section{RESEARCH METHOD}

\subsection{Research design}

This study was an observational, descriptive study with a cross-sectional design. The data collection is done retrospectively. Data were collected from medical records of cancer patients who had been admitted to the ICU of dharma's cancer special hospital (ICU of DCSH) in 2012-2014, medication administration records, sample submission, and culture examination report form.

\subsection{Subject}

We use the following criteria to recruit subjects, i.e. Adult patients who had nosocomial infections in the ICU; Patients with medical records in the ICU who received antibiotics in the 2012-2014 period. Patients originating from inpatients (wards); Patients with medical records were read. The exclusion criteria were postoperative patient and Patients with incomplete medical records.

\subsection{Research instruments}

We used a data collection form to collect secondary data from patient medical records, included name, Identification number, age, sex, date of hospital admission, hospital admission diagnosis, ICU admission date, ICU admission diagnosis, antibiotics used in hospitalization and ICU, bacterial culture data, vital signs (temperature, blood pressure, pulse, and respiration), laboratory data and patient radiology data.

\subsection{Data collecting procedure}

The list of cancer patients was obtained from a hospital computer database. The patients must be over the age of 18 years, treated in the ICU during 2012-2014, and receiving antibiotic therapy to be included in the study. They were excluded if they forced to return from the ICU, and their data was incomplete. Patient's medical records who had undergone treatment at the ICU of DCSH from 2012-2014 was selected and separated based on the inclusion and exclusion criteria by a trained health professional (pharmacists). Demographic (name, age, gender) data, the time and diagnosis when admitted to hospital and ICU, the time of the ICU discharge, the date of hospital discharge, the record of antibiotics use, and clinical data during hospitalization were taken from medical records. This study uses the definition of infection as determined by the USA central disease control (CDC) [6]. Infection is a condition (local or systemic), which is a reaction of the organism to the entry of or toxins released by infection-agent microorganisms. In this study, infection status is determined clinically based on the doctor's diagnosis, which was written in the medical record. Based on clinical manifestations and the doctor's diagnosis, the frequency in each patient is calculated. In contrast, we obtained drug use records from the nursing and pharmacy sections. Antibiotic use data from medical records were then matched with those from the nursing and pharmacy. Data on infectioncausing germs were taken from the submission and delivery forms of culture examination reports. Subsequently, the data were recorded on the data collection form and later transferred to the case report form. Only patients with a complete case report form were selected and designated as subjects. Recording of patient data on the case report form was done anonymously, but replaced with codes, to maintain patient confidentiality. 


\subsection{Ethical consideration}

The Dharmais Research ethics committee issued ethical clearance. The research protocol and informed consent have been reviewed and approved by the Dharmais Research ethics committee and the scientific committee of the Faculty of Pharmacy, Universitas Ahmad Dahlan.

\subsection{Data analysis}

We analyzed data descriptively in the form of percentages (frequency) for gender, age group, and cancer diagnosis. The antibiotics used are presented in rates by class and type.

\section{RESULTS AND DISCUSSION}

\subsection{Subject characteristic}

In the 2012-2014 period, 312 patients in the ICU were using antibiotics, but only 202 patients were eligible as research subjects. One hundred ten patients were excluded because of incomplete or missing medical records. Subjects' demographic data are presented in Table 1.

Table 1. Age, sex and cancer type of cancer patients in ICU

\begin{tabular}{|c|c|c|c|c|}
\hline \multicolumn{2}{|r|}{ Demographic Characteristics } & \multicolumn{3}{|c|}{ Year } \\
\hline \multicolumn{5}{|l|}{ age } \\
\hline & 18-25 years & $4(10.8)$ & $5(6.9)$ & $4(4.3)$ \\
\hline & $25-60$ years & $31(83.8)$ & $63(87.5)$ & $84(90.3)$ \\
\hline & $>60$ year & $2(5.4)$ & $4(5.6)$ & $5(5.4)$ \\
\hline Total & & $37(100)$ & $72(100)$ & $93(100)$ \\
\hline \multicolumn{5}{|l|}{ Sex } \\
\hline & Male & $23(61.2)$ & $23(31.9)$ & $37(39.8)$ \\
\hline & Female & $14(37.8)$ & $49(68.1)$ & $56(60.2)$ \\
\hline Total & & $37(100)$ & $72(100)$ & $93(100)$ \\
\hline \multicolumn{5}{|c|}{ Education } \\
\hline & Basic (elementary school-junior high school) & $4(10.8)$ & $8(11.1)$ & $17(18.3)$ \\
\hline & Advanced (senior high school-university) & $33(89.2)$ & $64(88.9)$ & $76(81.7)$ \\
\hline Total & & $37(100)$ & $72(100)$ & $93(100)$ \\
\hline \multicolumn{5}{|c|}{ Marriage status } \\
\hline & Married & $30(81.1)$ & $58(80.6)$ & $84(90.3)$ \\
\hline & Not married & $7(18.9)$ & $14(19.4)$ & $9(19.7)$ \\
\hline Total & & $37(100)$ & $72(100)$ & $93(100)$ \\
\hline \multicolumn{5}{|c|}{ Health payment } \\
\hline & Personal & $6(16.2)$ & $19(26.4)$ & $12(12.9)$ \\
\hline & Institution & $8(21.6)$ & $9(12.5)$ & $8(8.6)$ \\
\hline & $J K N$ insurance & $23(62.2)$ & $44(61.10)$ & $73(78.5)$ \\
\hline Total & & $37(100)$ & $72(100)$ & $93(100)$ \\
\hline \multicolumn{5}{|l|}{ Job } \\
\hline & Labor & $10(27.0)$ & $35(48.6)$ & $45(48.4)$ \\
\hline & Employed by the government or private sectors & $27(73.0)$ & $37(51.4)$ & $48(51.6)$ \\
\hline Total & & $37(100)$ & $72(100)$ & $93(100)$ \\
\hline
\end{tabular}

The number of cancer patients treated in the ICU of DCSH, Jakarta from 2012-2014, who met the inclusion criteria was 202. The number of cancer patients tends to increase every year. Descriptive analysis of the patients' characteristics shows that the number of male patients was lower than that of females, with $83(41.1 \%)$ and $119(58.9 \%)$, respectively. In terms of age, the majority of patients were between 18 and 45 years with a total of 104 patients (51.5\%), while patients with senior high school-university education outnumbered those with junior high school or elementary school (82\% compared to $12 \%$ ). Based on the age, gender, and educational background, it is known that most cancer patients who require treatment with antibiotics in the ICU were productive age (25-65years) women with senior high school-university education. This finding not only illustrates that women in the fertile period seem to be more susceptible to infection, but it also shows that the level of health in Indonesia is still generally low. The study revealed that the public health level of productive age in Indonesia is below the government's targets and expectations. Promotive and preventive efforts are required primarily to increase awareness, willingness, and ability of the public to engage in a healthy lifestyle to avoid cancer from an early age as an investment for the development of human resources who are socially and economically productive [21, 22].

\subsection{Clinical characteristics}

Table 2 present the primary diagnosis and the patients' clinical symptoms. We can see that most of the patients were diagnosed with leukemia and mammary cancer, and most of them $(>50 \%)$ were treated in the ICU for less than fifteen days, with only a small percentage staying in the ICU for more than 15 days. 
Based on the data, patients who most frequently suffered from infections were blood cancers (leukemia), lung cancer, and cervical cancer. In contrast, the most common types of diseases that affected The patients were lung infection (pneumonia), central venous infections, urinary tract infections, and wounds. The results are consistent with the general pattern of cancer in Indonesia and the type of infection in ICU $[23,24]$.

The group and type of antibiotics used for the treatment of infections also fit the microorganism pattern in Indonesia. Cancer patients given antibiotics in the ICU were constantly increasing from 2012 s.d. 2014. In 2012, 37 patients received antibiotic therapy. The figure nearly doubled in 2013 with 72 patients and continued to grow to 92 patients in 2014. Based on the cancer types, most patients had leukemia, mammary cancer, and cervical cancer. The patients were given antibiotics due to pneumonia (52\%-59\%), central infection (20\%-26\%), urinary tractus infection UTI (11\%-19\%) and wounds (1\%-3\%). Most of them suffered from more than one infection with an average of more than one infection incidence (1.24 in 2012, 1.1 in 2013, and 1.25 in 2014). The pattern of infection events in the DCSH ICU is consistent with the pattern of infections in the ICU of previous studies [3, 24, 25].

Table 2. The primary and additional diagnosis of antibiotic use indications among the cancer patients

\begin{tabular}{|c|c|c|c|}
\hline Clinical characteristics & \multicolumn{3}{|c|}{ Year } \\
\hline Type of Cancer Diagnosis & & & \\
\hline Leukemia (AML, ALL, CLL, LGK) & $7(18.9)$ & $11(15.3)$ & $23(24.7)$ \\
\hline Mammae cancer & $5(13.5)$ & $13(18.1)$ & $17(18.3)$ \\
\hline Servix cancer & $1(2.7)$ & $7(9.7)$ & $11(11.8)$ \\
\hline Non-Hodgkin lymphoma & $2(5.4)$ & $10(13.9)$ & $7(7.5)$ \\
\hline Lung cancer & $7(18.9)$ & $6(8.3)$ & $6(6.5)$ \\
\hline Ovary cancer & $1(2.7)$ & $2(2.8)$ & $4(4.3)$ \\
\hline Nasopharynx cancer & $0(0)$ & $5(6.9)$ & $2(2.2)$ \\
\hline Thyroid cancer & $1(2.7)$ & $4(5.6)$ & $1(1.1)$ \\
\hline Colon cancer & $0(0)$ & $1(1.4)$ & $5(5.4)$ \\
\hline Abdomen cancer & $0(0)$ & $4(5.6)$ & $4(4.3)$ \\
\hline Frontal tumor & $3(8.1)$ & $0(0)$ & $4(4.3)$ \\
\hline Tongue cancer & $1(2.7)$ & $1(1.4)$ & $1(1.1)$ \\
\hline Prostat \& testis cancer & $3(8.1)$ & $1(1.40)$ & $0(0)$ \\
\hline Astrochytoma & $2(5.4)$ & $1(1.4)$ & $1(1.1)$ \\
\hline Others & $4(10.8)$ & $7(7.4)$ & $7(7.7)$ \\
\hline Total & $37(100)$ & $72(100)$ & $93(100)$ \\
\hline \multicolumn{4}{|l|}{ Leukocytosis status in the ICU } \\
\hline Leukocytosis & $26(70.3)$ & $50(69.4)$ & $65(69.9)$ \\
\hline Not leukocytosis & $11(29.7)$ & $22(30.6)$ & $28(30.1)$ \\
\hline Total & $37(100)$ & $72(100)$ & $93(100)$ \\
\hline \multicolumn{4}{|l|}{ Infection diagnosis } \\
\hline Pneumonia & $24(52.2)$ & $47(59.5)$ & $69(59.5)$ \\
\hline UTI & $9(19.6)$ & $9(11.4)$ & $21(18.1)$ \\
\hline Infection central & $12(26.1)$ & $20(25.3)$ & $24(20.6)$ \\
\hline Wound & $1(2.2)$ & $3(3.8)$ & $2(1.8)$ \\
\hline Total & $46(100)$ & $79(100)$ & $116(100)$ \\
\hline Mean of infection diagnosis per patients & 1.24 & 1.1 & 1.25 \\
\hline \multicolumn{4}{|l|}{ Duration in ICU care } \\
\hline$<5$ day & $24(64.9)$ & $37(51.4)$ & $60(64.5)$ \\
\hline 5-15 day & $10(27.0)$ & $24(33.3)$ & $24(25.8)$ \\
\hline$>15$ day & $3(8.1)$ & $11(15.3)$ & $9(9.7)$ \\
\hline Total & $37(100)$ & $72(100)$ & $93(100)$ \\
\hline
\end{tabular}

\subsection{Use of group-based antibiotics}

The group of antibiotics $(\mathrm{AB})$ prescribed to the patients and their various indications from 2012 to 2014 are presented in Table 3. Meropenem was the most frequently prescribed among all antibiotics accounted for $27.3 \%$ in $2012,29.5 \%$ in 2013, and $38.6 \%$ in 2014. During the three years, meropenem prescription reached as high as $33.8 \%$ of the total antibiotic prescriptions for infections in cancer patients. Research data also shows that there were fluctuations in the number of antibiotic types prescribed to the patients with 20,26, and 23 types for 2012, 2013, and 2014 respectively. The pattern of antimicrobial use in the DCSH ICU is under the pattern of antibiotic use as a result of research in Lebanon [20], China [4], and Korea [23], which is experiencing fluctuations in the number and type of antibiotics. From the data in Table 3, it is known that the use of antibiotics in Indonesia is more than the use of antibiotics in the ICU from previous studies, which is more than 20 types of antibiotics. The last report research stated that the average consumption of antibiotics in the ICU was 20 types $[18,26]$. Of the 202 patients admitted 
to the ICU, most patients used more than one type of antibiotics. Following Table 2, it is known that most subjects have more than one type of infection. Such antibiotic combination usage can be justified in the following circumstances: initial treatment of patients with severe diseases, in polymicrobial infections to prevent the onset of resistant microorganisms, and to obtain a synergistic effect [25, 27].

Table 3. Group and type of antibiotics prescribed to the cancer patients

\begin{tabular}{|c|c|c|c|}
\hline \multirow{2}{*}{ Level and Type of Antibiotics } & \multicolumn{3}{|c|}{ Year } \\
\hline & $2012 \mathrm{n}(\%)$ & $2013 \mathrm{n}(\%)$ & $2014 \mathrm{n}(\%)$ \\
\hline \multicolumn{4}{|l|}{ Cephalosporin 3th Generation group } \\
\hline Ceftazidim & $1(1.5)$ & $6(4.5)$ & $3(1.8)$ \\
\hline Cefotaxime & $2(3.0)$ & $3(2.3)$ & $3(1.8)$ \\
\hline Ceftizoxim & $1(1.5)$ & $2(1.5)$ & $0(0)$ \\
\hline Cefoperazon & $2(3.0)$ & $7(5.3)$ & $10(5.8)$ \\
\hline Ceftriaxon & $7(\mathbf{1 0 . 6})$ & 14 (10.6) & $15(8.8)$ \\
\hline \multicolumn{4}{|l|}{ Cephalosporin 4th Generation groups } \\
\hline Cefpirom & $2(3.0)$ & $1(0.8)$ & $2(1.2)$ \\
\hline Cefepim & $0(0)$ & $3(2.3)$ & $4(2.3)$ \\
\hline \multicolumn{4}{|c|}{ Combination of cephalosporin 3th Generation } \\
\hline Sulbactam-Cefoperazon & $2(3.0)$ & $4(3.0)$ & $4(2.3)$ \\
\hline \multicolumn{4}{|l|}{ Carbapenem groups } \\
\hline Meropenem & $18(27.3)$ & $39(29.5)$ & $66(38.6)$ \\
\hline Doripenem & $3(4.5)$ & $4(3.0)$ & $0(0)$ \\
\hline \multicolumn{4}{|l|}{ Aminoglycoside group } \\
\hline Amikacin & $4(6.1)$ & $5(3.8)$ & $8(4.7)$ \\
\hline Gentamicin & $1(1.5)$ & $2(1.5)$ & $0(0)$ \\
\hline Streptomycin & $0(0)$ & $1(0.8)$ & $0(0)$ \\
\hline Netilmicin & $0(0)$ & $2(1.5)$ & $0(0)$ \\
\hline \multicolumn{4}{|l|}{ Fluoroquinolone group } \\
\hline Levofloxacin & $5(7.6)$ & $23(17.4)$ & $26(15.2)$ \\
\hline Ciprofloxacin & $2(3.0)$ & $1(0.8)$ & $6(3.5)$ \\
\hline Ofloxacin & $2(3.0)$ & $0(0)$ & $0(0)$ \\
\hline Moxifloxacin & $0(0)$ & $0(0)$ & $2(1.2)$ \\
\hline \multicolumn{4}{|l|}{ Glycycline group } \\
\hline Tigecycline & $2(3.0)$ & $1(0.8)$ & $2(1.2)$ \\
\hline \multicolumn{4}{|l|}{ Derivat Imidazole group } \\
\hline Metronidazole & $3(4.5)$ & $1(0.8)$ & $6(3.5)$ \\
\hline \multicolumn{4}{|l|}{ Glycopeptide group } \\
\hline Teicoplanin & $0(0)$ & $1(0.8)$ & $1(0.6)$ \\
\hline \multicolumn{4}{|l|}{ Penicilin+ Beta-Lactamase Inhibitor group } \\
\hline Amoxicillin+Klavulanat Acid & $0(0)$ & $1(08)$ & $0(0)$ \\
\hline Tazobactam+Piperacilin & $7(\mathbf{1 0 . 6})$ & $6(45)$ & $10(5.8)$ \\
\hline \multicolumn{4}{|l|}{ Penicillin group derivates } \\
\hline Amoxicillin & $0(0)$ & $0(0.0)$ & $1(0.6)$ \\
\hline \multicolumn{4}{|l|}{ Other Antibiotic } \\
\hline Linezolide & $1(1.5)$ & $4(3.0)$ & $2(1.2)$ \\
\hline Fosfomycin & $0(0)$ & $1(0.8)$ & $0(0)$ \\
\hline Total & $66(100.0)$ & $132(100.0)$ & $171(100.0)$ \\
\hline
\end{tabular}

The pattern of antibiotic use in hospitals is influenced by many factors, including germ distribution patterns, clinical conditions, types of services, and extraordinary conditions (epidemics) [28-29]. Covid-19 pandemic influences changes in disease patterns and the use of anti-infective drugs, including antibiotics in cancer patients in hospitals, including in the emergency room, wards, and in the ICU [30]. Cancer patients are one of the vulnerable groups in the COVID-19 pandemic, besides diabetics, hypertension, and cardiovascular disease, and dyslipidemic disease. Based on the results of the latest research, it is known that the top three most prevalence of cancer patients with the need to undergo treatment in hospital are lung cancer, breast cancer, and colorectal cancer [31]. The pattern of hospital visits with cancer patients' research datain the pandemic phase is almost the same as the pattern of hospital visits of patients in the non-pandemic era, but not so with the use of anti-infective drugs. Research in Wuhan and Hubei showed that the highest incidence of infection in cancer patients treated in the ICU was pneumonia due to SARS COV-2 infection with septic shock. The main anti-infective drugs for patient care in the ICU are antivirals, and more than $84 \%$ of patients get intravenous antibiotics [32-33]. Germs that cause lung infections in the COVID-19 pandemic are viruses, in contrast to non-pandemic conditions where the most significant cause of pneumonia in cancer patients is bacteria. 
The pattern of using antibiotics results of this study when compared with the pattern of antibiotics use of cancer patients in the emergency department (ED) there are differences. Patients cancer in the ED received beta-lactam (86.7\%) anti-pseudomonal (13.6\%), vancomycin aminoglycosides (9.6\%) and carbapenem (3.2\%). The pattern of the incidence of infection in cancer patients in ICU-DCSH is slightly different from the prevalence of infection in cancer patients from the results of a recent study conducted in the ED. The results of the study of the incidence of infections in cancer patients treated in the ED were mostly respiratory infections, then followed by skin infections and digestive tract infections. At the same time, the incidence of infection in cancer patients in DCSH is pneumonia, then followed by central infection and urinary tract infections [34].

\section{CONCLUSION}

Meropenem is the most commonly prescribed antibiotic for cancer patients in the ICU of DCSH, followed by levofloxacin and ceftriaxone. An increase in prescribing antibiotics meropenem, ceftriaxone, and levofloxacin from 2012-2014. Most infectious diseases that use antibiotics in the ICU of DCSH are pneumonia, UTI, central infection, and wound.

\section{ACKNOWLEDGEMENTS}

Researchers thank and appreciate all volunteers, ICU staff, and Dharmais National Cancer Special Hospital Directors for the opportunities and facilities so that the study can be completed. The author also gives appreciation and profound thanks to Dr. Dra. Rizka Andalusia, M. Pharm, MARS., Apt., Who has provided guidance, support, and facilities in data retrieval at the Medical Record Installation and in the Pharmacy Installation at the Dharmais Cancer Special Hospital.

\section{REFERENCES}

[1] Seok H., Ko J.-H., Peck K. R., Kim J.-Y., Lee J. H., Park G. E., et al., "Treatment of Community-onset Pneumonia in Neutropenic Cancer Patients," Pneumonia, vol. 11, no. 2, 2019.

[2] Anggriani Y., Banun A. E., "Evaluation of Antibiotic Usage in HCU and ICU Wards in "Dharmais" Cancer Hospital February to March 2012," Indonesian Journal of Pharmacy Science, pp. 182-190, 2013.

[3] Yunus L., Prihartono N. A. T. D., "Primary Infection of Blood Flow Patient with Cancer associated with central venous catheter at RSK "Dharmais"," in 2011- 2012, 2013.

[4] Zhang Y., Yao Z., Zhan S., Yang Z., Wei D., Zhang J., et al., "Disease burden of intensive care unit-acquired pneumonia in China: A systematic review and meta-analysis," International Journal of Infectious Diseases, vol. 29, pp. 84-90, 2014.

[5] Baditoiu L., Axente C., Lungeanu D., Muntean D., Horhat F., Moldovan R., et al., "Intensive care antibiotic consumption and resistance patterns: A cross-correlation analysis," Annals of Clinical Microbiology and Antimicrobials, vol. 16, no. 71, 2017.

[6] Al-Abdely H. M., Khidir Mohammed Y., Rosenthal V. D., Orellano P. W., ALazhary M., Kaid E., et al., "Impact of the International Nosocomial Infection Control Consortium (INICC)'s multidimensional approach on rates of ventilator-associated pneumonia in intensive care units in 22 hospitals of 14 cities of the Kingdom of Saudi Arabia," Journal of Infection and Public Health, vol. 11, no. 5, pp. 677-684, 2018.

[7] Fourie T., Schellack N., Bronkhorst E., Coetzee J., Godman B., "Antibiotic prescribing practices in the presence of extended-spectrum $\beta$-lactamase (ESBL) positive organisms in an adult intensive care unit in South Africa-A pilot study," Alexandria Journal of Medicine, vol. 54, no. 4, pp. 541-547, 2018.

[8] Parikh P., Bhat V., "Urinary tract infection in cancer patients in a tertiary cancer setting in India: microbial spectrum and antibiotic susceptibility pattern," Antimicrobial Resistance and Infection Control, vol. 4, no. P221, 2015.

[9] De Oliveira T. F. L., Filho I. S. G., Passos J. de S., da Cruz S. S., Oliveira M. T., Trindade S. C., et al., "Factors associated with nosocomial pneumonia in hospitalized individuals," Revista da Associacao Medica Brasileira, vol. 57, no. 6, pp. 630-636, 2011.

[10] Su B. C., Lin C. C., Su C. W., Hui Y. L., Tsai Y. F., Yang M. W., et al., "Ultrasonic cardiac output monitor provides accurate measurement of cardiac output in recipients after liver transplantation," Acta Anaesthesiologica Taiwanica, vol. 46, no. 4, pp. 171-177, 2008.

[11] Perron T., Emara M., Ahmed S., "Time to antibiotics and outcomes in cancer patients with febrile neutropenia," $B M C$ Health Services Research, vol. 14, no. 162, 2014.

[12] Rhedin S., Galanis I., Granath F., Ternhag A., Hedlund J., Spindler C., et al., "Narrow-spectrum B-lactam monotherapy in hospital treatment of community-acquired pneumonia: a register-based cohort study," Clinical Microbiology and Infection, vol. 23, no. 4, pp. 247-252, 2017.

[13] Bozkurt F., Kaya S., Tekin R., Gulsun S., Deveci O., Dayan S., et al., "Analysis of antimicrobial consumption and cost in a teaching hospital,” Journal of Infection and Public Health, vol. 7, no. 2, pp. 161-169, 2014.

[14] Schellack N., Benjamin D., Brink A., Duse A., et al., "A situational analysis of current antimicrobial governance, regulation, and utilization in South Africa," International Journal of Infectious Diseases, vol. 64, pp. 100-106, 2017. 
[15] Feng D. Y., Zhou Y. Q., Zou X. L., et al., "Factors influencing mortality in hospital-acquired pneumonia caused by Gram-negative bacteria in China," Journal of Infection and Public Health, vol. 12, no. 5, pp. 630-633, 2019.

[16] Falcone M., Venditti M., Shindo Y., Kollef M. H., "Healthcare-associated pneumonia: Diagnostic criteria and distinction from community-acquired pneumonia," International Journal of Infectious Diseases, vol. 15, no. 8, pp. e545-550, 2011.

[17] García-Rey C., Martín-Herrero J. E., Baquero F., "Antibiotic consumption and generation of resistance in Streptococcus pneumoniae: The paradoxical impact of quinolones in a complex selective landscape," Clinical Microbiology and Infection, vol. 12, pp. 55-66, 2006.

[18] Saxena S., Priyadarshi M., Saxena A., Singh R., "Antimicrobial consumption and bacterial resistance pattern in patients admitted in I.C.U at a tertiary care center," Journal of Infection and Public Health, vol. 12, no. 5, pp. 695-699, 2019.

[19] Leone M., Bouadma L., Bouhemad B., Brissaud O., Dauger S., Gibot S., et al., "Hospital-acquired pneumonia in ICU," Anaesthesia Critical Care and Pain Medicine, vol. 37, no. 1, pp. 83-98, 2018.

[20] Iskandar K., Hanna P. A., Salameh P., Raad E. B., "Antibiotic consumption in non-teaching Lebanese hospitals: A cross-sectional study," Journal of Infection and Public Health, vol. 9, no. 5, pp. 618-625, 2016.

[21] Narain J. P., Garg R., Fric A., "Non-communicable diseases in the South-East Asia Region: Burden, strategies and opportunities," The National Medical Journal of India, vol. 24, no. 5, pp. 280-287, 2011.

[22] Edition F., "Global Cancer," p. 700, 2008.

[23] Park H. K., Song J. U., Um S. W., Koh W. J., Suh G. Y., Chung M. P., et al., "Clinical characteristics of health care-associated pneumonia in a Korean teaching hospital," Respiratory Medicine, vol. 104, vol. 11, pp. 1729-1735, 2010.

[24] Adysaputra S. A., Rauf A. M. B. B., "Pattern of surgical wound germs in Intensive Care Unit Wahidin Sudirohusodo Hospital,” Indonesian Journal on Medical Science, vol. 2, no. 2, pp. 67-70, 2009.

[25] Liang C. A., Lin Y. C., Lu P. L., Chen H. C., Chang H. L., Sheu C. C., "Antibiotic strategies and clinical outcomes in critically ill patients with pneumonia caused by carbapenem-resistant Acinetobacter baumannii," Clinical Microbiology and Infection, vol. 24, no. 8, pp. 908.e1-908.e7, 2018.

[26] Axente C., Licker M., Moldovan R., Hogea E., Muntean D., Horhat F., et al., "Antimicrobial consumption, costs and resistance patterns: A two year prospective study in a Romanian intensive care unit," BMC Infectious Diseases, vol. 17 , no. $358,2017$.

[27] Alotair H. A., Hussein M. A., Elhoseny M. A., Alzeer A. H., Khan M. F., "Severe pneumonia requiring ICU admission: Revisited,” Journal of Taibah University Medical Science, vol. 10, no. 3, pp. 293-299, 2015.

[28] U. Hadi et al., "Audit of antibiotic prescribing in two governmental teaching hospitals in Indonesia," Clin. Microbiol. Infect., vol. 14, no. 7, pp. 698-707, 2008.

[29] G. S. Guven and O. Uzun, "Principles of good use of antibiotics in hospitals," J. Hosp. Infect., vol. 53, no. 2, pp. 91-96, 2003.

[30] V. C. C. Cheng et al., "Escalating infection control response to the rapidly evolving epidemiology of the Coronavirus disease 2019 (COVID-19) due to SARS-CoV-2 in Hong Kong," Infect. Control Hosp. Epidemiol., vol. 2019, pp. 493-498, 2020.

[31] A. Addeo and A. Friedlaender, "Cancer and COVID-19: Unmasking their ties," Cancer Treat. Rev., vol. 88, no. May, p. 102041, 2020.

[32] K. Yang et al., "Clinical characteristics, outcomes, and risk factors for mortality in patients with cancer and COVID-19 in Hubei, China: a multicentre, retrospective, cohort study," Lancet Oncol., vol. 2045, no. 20, pp. 1-10, 2020.

[33] L. Zhang et al., "Clinical characteristics of COVID-19-infected cancer patients: a retrospective case study in three hospitals within Wuhan, China," Ann. Oncol., vol. 31, no. 7, 2020.

[34] O. Peyrony et al., "Antibiotic prescribing and outcomes in cancer patients with febrile neutropenia in the emergency department," PLoS One, vol. 15, no. 2, p. e0229828, 2020. 